\title{
Optimized Power Allocation and Relay Location Selection in Cooperative Relay Networks
}

\author{
Jianrong Bao, ${ }^{1,2}$ Jiawen Wu, ${ }^{1}$ Chao Liu, ${ }^{1}$ Bin Jiang, ${ }^{1}$ and Xianghong Tang ${ }^{1}$ \\ ${ }^{1}$ School of Communication Engineering, Hangzhou Dianzi University, Hangzhou 310018, China \\ ${ }^{2}$ National Mobile Communications Research Laboratory, Southeast University, Nanjing 210096, China \\ Correspondence should be addressed to Bin Jiang; jiangbin@hdu.edu.cn
}

Received 4 April 2017; Revised 2 July 2017; Accepted 11 October 2017; Published 9 November 2017

Academic Editor: Haiyu Huang

Copyright (c) 2017 Jianrong Bao et al. This is an open access article distributed under the Creative Commons Attribution License, which permits unrestricted use, distribution, and reproduction in any medium, provided the original work is properly cited.

\begin{abstract}
An incremental selection hybrid decode-amplify forward (ISHDAF) scheme for the two-hop single relay systems and a relay selection strategy based on the hybrid decode-amplify-and-forward (HDAF) scheme for the multirelay systems are proposed along with an optimized power allocation for the Internet of Thing (IoT). Given total power as the constraint and outage probability as an objective function, the proposed scheme possesses good power efficiency better than the equal power allocation. By the ISHDAF scheme and HDAF relay selection strategy, an optimized power allocation for both the source and relay nodes is obtained, as well as an effective reduction of outage probability. In addition, the optimal relay location for maximizing the gain of the proposed algorithm is also investigated and designed. Simulation results show that, in both single relay and multirelay selection systems, some outage probability gains by the proposed scheme can be obtained. In the comparison of the optimized power allocation scheme with the equal power allocation one, nearly 0.1695 gains are obtained in the ISHDAF single relay network at a total power of $2 \mathrm{~dB}$, and about 0.083 gains are obtained in the HDAF relay selection system with 2 relays at a total power of $2 \mathrm{~dB}$.
\end{abstract}

\section{Introduction}

Recently, multiple-input multiple-output (MIMO), as a milestone in the development of wireless communications, brought an efficient transmission rate and reliability. To put it into practice, a cooperative communication scheme was then proposed in time [1], and it had been widely used and rapidly developed. In cooperative communications, the diversity gain was obtained, when the relay node forwarded messages and the destination node combined the received signals from both the source and relay nodes. According to different strategies for processing signals at the relay nodes, there are mainly three cooperation schemes, such as the amplify-and-forward (AF) [1], the decode-and-forward (DF) [2], and the coded cooperation (CC) [3]. To solve the deficiency of AF relay amplifying both the noises and signals, causing the incorrect DF relay decoding and also the error propagation phenomenon, an incremental relay protocol [4], accompanied by a hybrid decode-amplify-andforward (HDAF), was proposed [5]. For the shortage of the incremental relay protocol, the incremental selection amplify-and-forward (ISAF) [6] was investigated, which selected the proper occasion to retransmit the messages in the source according to the channel estimation, when the direct transmission between the source and destination was failed. But the noise amplification still remained. Then, an incremental selection hybrid decode-amplify forward (ISHDAF) scheme was proposed in [7], where the HDAF scheme was combined with incremental selection strategy. Compared with the aforementioned ISAF scheme, the ISHDAF scheme had a significant improvement in bit error rate (BER) and outage probability, since both the BER and outage probability of a cooperative transmission system in the DF strategy were lower than those in the AF strategy. According to the principle of incremental relay, the average spectral efficiency of the ISHDAF scheme was also higher than that of the HDAF scheme. However, all gains were obtained under equal power allocation of the source and relay nodes for the low systematic complexity, which caused the deficiency of only few performance improvements. To improve the 
spectral efficiency of the system, there was also a signal-tonoise ratio (SNR) based incremental hybrid decode-amplify forward (IHDAF) protocol proposed in [8]. Furthermore, the SNR thresholds, the power allocation schemes, and the relay locations were studied to optimize the outage probability and BER performance.

Meanwhile, power allocation in cooperation communications had always been one of the research hot-spots. In wireless uplink transmissions, successive interference cancellation (SIC) was combined with the power allocation method to obtain the optimal power allocation ratio for efficient resource allocation [9]. For relay forwarding systems, two power allocation methods, by the Lagrange multiplier method and the differential algorithm, respectively, were also proposed for the lower bound of symbol error rate (SER) in the HDAF relay cooperative networks [10]. Xiao and Ouyang in [11] developed a two-source-destination-pair cooperative network with the HDAF protocol. And a closedform expression of the outage probability was derived and thus a minimal total power was obtained under the constraints of the supposed outage probability. Similarly, for the two-source-destination-pair system, there was also a parallel shift water filling algorithm proposed for the power allocation [12]. The advantage of it over the conventional ones was the reduced complexity by just eliminating the iterative searching process. However, the cost is a little performance decrease. In [13], a multirelay selection scheme with joint power allocation was proposed, featured with the significantly decreased complexity of computation. To achieve this effect, it used a simple power reallocation during the multirelay selection process. Also a joint relay selection and power allocation scheme for cooperative wireless sensor networks was proposed in [14]. It adaptively chose the proper relays and their transmission power to maximize the signal-to-noise ratio (SNR) at the destination by the channel state information (CSI). Then a SNR-based relay selection for IHDAF cooperative diversity protocol was also proposed in [15], and the closed-form expressions of average channel capacity and outage probability were derived simultaneously. Also a swarm intelligencebased power allocation and relay selection algorithm could be used for wireless cooperative networks [16]. It could not only reduce the computational complexity effectively, but also select the optimal relay nodes to solve the nonlinear optimization problems by a fast global search with low cost. In [17], with a AF protocol based two-hop multiple energyharvesting relays network, an improved power allocation was investigated to improve the whole outage performance. The innovation in the proposed scheme lied in the fact that it jointly maximized the transmit power under the constraints of limited individual relay energy. Also the power allocation and relay selection strategies in both dual-hop and multihop scenarios in cognitive relay networks were researched [18]. They achieved the good features of both minimal total transmit power and maximal entire network capacity. For the relay selection optimization, there had been a dynamic strategy to choose the best relay node and path under the constraints of total power and power allocation for each relay [18]. In addition, an improved relay selection strategy of HDAF scheme was proposed to improve the BER and outage probability [19]. It can adaptively select the AF or DF forward strategy for all relays according to the channel quality. Then the best relay was chosen to forward signals. However, it still used the equal power allocation to reduce the complexity. In [20], a power allocation algorithm by the lowest average bit error was proposed for these infrastructure-less networks using unbalanced communication links. To investigate the influence of the links on the system performance, the location of the relay node with respect to the source and destination nodes was also studied. Unfortunately, only the effect of certain node locations, rather than the optimal relay location, was determined. Subsequently, a much more detailed study about the optimal relay location was presented in [21], but under simply fixed ratio nodes power.

In this paper, by analyzing a two-hop single relay cooperative network with an ISHDAF scheme and the multirelay selection strategy with a HDAF scheme, an optimized power allocation is proposed. The main contributions are summarized as follows:

(1) The power allocation is optimized in both the ISHDAF single relay and the HDAF multirelay systems. In the case of link status change, the preferred links are allocated with much more power for transmission according to the well-known water filling principle in information theory, which reduces the entire power consumption under the same system performance. The proposed scheme also provides a new hybrid automatic repeat request (ARQ) retransmission and relay forward mechanism, where the source node can retransmit messages to the destination node when the first direct transmission failed. It differs from the source node sending new messages to the destination node directly in the incremental relaying protocol. So it can be more suited for all kinds of the multiple relay channel status and obviously improves the systematic outage probability without any complexity increase.

(2) The approximate closed-form expression of the systematic outage probability with relation to the node power and channel coefficients is derived by the equivalent infinitesimal replacement of the probability distribution function at high SNR. And it can be taken as the objective function of the optimization. Then, the minimization is achieved under fixed total power by Lagrange multiplier method, and the objective function is related to the power of the source node and the relay nodes. The power allocation coefficients between the source and the relay nodes are then obtained to achieve optimized power allocation. Moreover, the power allocation changes the location selection of the relay nodes, which can be calculated indirectly from the above closed-form expression. It can adaptively satisfy the link conditions to optimize the entire system performance.

(3) By introducing the path loss factor, the powers of the source and the relay nodes are modeled as the objective function related to the distance among all nodes. According to both the property of the objective 


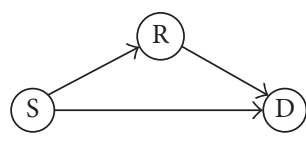

FIGURE 1: System model of a single relay communication.

function and the related numerical analyses, the relationships of the varied power to the distance of the nodes are obtained. Then, the optimized node positions are obtained to improve the power efficiency with minimized systematic outage probability. Also the power allocation of all relay nodes with respect to their relative location to the source and destination nodes can be clearly and quantitatively analyzed by this model. Therefore, the link status associated with the proposed relay position obviously affects the selection of the cooperative schemes, which can be adopted in practice.

This paper is organized as follows. In Section 2, a single relay cooperation system with the ISHDAF scheme is introduced. Section 3 presents a multirelay selection strategy based on the HDAF scheme. Subsequently, the analytical expressions of the outage probability of both the ISHDAF and the HDAF relay system are derived in Section 4. In this section, an optimized power allocation using Lagrange Method is also proposed to minimize the outage probability. Simultaneously, the close-form analytical expression of the optimal relay location of the proposed algorithm is given to manifest the relationship between the relay location and the outage probability. After that, in Section 5, the simulation results and analyses are presented to verify the good outage probability and optimal power allocation brought by the proposed algorithm. The optimal relay location by the proposed method is also given and tested to be effective. Finally, Section 6 concludes the whole paper.

\section{Single Relay Model and ISHDAF Mutual Information Evaluation}

For a classic three-node relay model shown in Figure 1, it consists of a source node $S$, a relay node $\mathrm{R}$, and a destination node $\mathrm{D}$. Equipped with a single omnidirectional antenna, all nodes communicates with each other. The ideal channel state information (CSI) can be obtained through channel training. For the independent links S-D, S-R, and R-D, their channel gains, that is, $\left|h_{s d}\right|^{2},\left|h_{s r}\right|^{2}$, and $\left|h_{r d}\right|^{2}$, are subject to the exponential distribution with channel parameters as $1 / \sigma_{s d}{ }^{2}, 1 / \sigma_{s r}{ }^{2}$, and $1 / \sigma_{r d}{ }^{2}$, respectively. At a flat Rayleigh fading channel, the noise is an additive white Gaussian noise (AWGN), with zero mean and variance $N_{0}$.

In an ISHDAF cooperative network, the whole transmission is divided into two time slots. In the first slot, node $S$ sends a signal to node $\mathrm{R}$ and node $\mathrm{D}$, while in the second slot, either node $S$ or node $R$ sends the signal to node $D$, which depends on the link status. Suppose that the transmitted power of the source node $S$ is $P_{S 1}$, and the information transmission rate is $R \mathrm{bit} / \mathrm{s}$. There are two main situations according to the decoding results of the destination node.

For the first situation, if the destination node successfully receives the signal sent by the source in the first slot, the transmission from node $\mathrm{S}$ to node $\mathrm{D}$ is not interrupted. In this case, the mutual information is defined in [22] as

$$
I_{\mathrm{DT}}=\frac{1}{2} \cdot \log _{2}\left(1+\frac{\left|h_{s d}\right|^{2} P_{S 1}}{N_{0}}\right),
$$

which needs to be larger than $R$ according to the information theory. By simplifying (1) and the condition of $I_{\mathrm{DT}}>R$, the relationship of $\left|h_{s d}\right|^{2}>\left(2^{2 R}-1\right) N_{0} / P_{S 1}$ is obtained. Given the threshold as $T_{1}=\left(2^{2 R}-1\right) N_{0} / P_{S 1}$, and $\left|h_{s d}\right|^{2}>T_{1}$, the source node $\mathrm{S}$ keeps transmitting directly to the destination node $\mathrm{D}$ in the second slot, and the relay node $\mathrm{R}$ remains inactive.

For another situation, if the direct transmission fails in the first slot, or the destination does not receive the correct information from the source, the source would retransmit the message to the destination in the second slot. In this case, the mutual information is deduced and presented in [6] as

$$
I_{\mathrm{DRT}}=\frac{1}{2} \cdot \log _{2}\left(1+\frac{2\left|h_{s d}\right|^{2} P_{S 1}}{N_{0}}\right) .
$$

To ensure the success retransmission, (2) should also be larger than $R$ and it can obtain $\left|h_{s d}\right|^{2}>T_{1} / 2$. When $T_{1} / 2<$ $\left|h_{s d}\right|^{2} \leq T_{1}$, the source retransmits message and the relay remains inactive in the second slot.

The relay node starts the cooperative transmission when there is $\left|h_{s d}\right|^{2} \leq T_{1} / 2$. Then, if the relay can correctly decode the information from the source, the DF scheme is adopted in the second slot. It needs to satisfy the relationship of $1 / 2$. $\log _{2}\left(1+\left|h_{s r}\right|^{2} P_{S 1} / N_{0}\right)>R$, or $\left|h_{s r}\right|^{2}>T_{1}$, which is the required condition for the relay to forward the correct signal in the DF protocol. Otherwise, when $\left|h_{s r}\right|^{2} \leq T_{1}$, the AF protocol is used instead.

If the DF scheme is adopted for the cooperative transmission, the mutual information is obtained by the maximum ratio combining (MRC) for the destination as

$$
I_{\mathrm{DF}}=\frac{1}{2} \cdot \log _{2}\left(1+\frac{\left|h_{s d}\right|^{2} P_{S 1}}{N_{0}}+\frac{\left|h_{r d}\right|^{2} P_{R 1}}{N_{0}}\right) .
$$

If the relay node transmits in the AF protocol, the mutual information is expressed in [6] as

$$
\begin{aligned}
I_{\mathrm{AF}} & =\frac{1}{2} \cdot \log _{2}\left[1+\frac{\left|h_{s d}\right|^{2} P_{S 1}}{N_{0}}\right. \\
& \left.+f\left(\frac{\left|h_{s r}\right|^{2} P_{S 1}}{N_{0}}, \frac{\left|h_{r d}\right|^{2} P_{R 1}}{N_{0}}\right)\right],
\end{aligned}
$$

where $f(x, y)=x y /(1+x+y)$.

In summary, the mutual information in the ISHDAF cooperative network is concluded as follows. When $\left|h_{s d}\right|^{2}>T_{1}$, the source directly transmits to the destination 


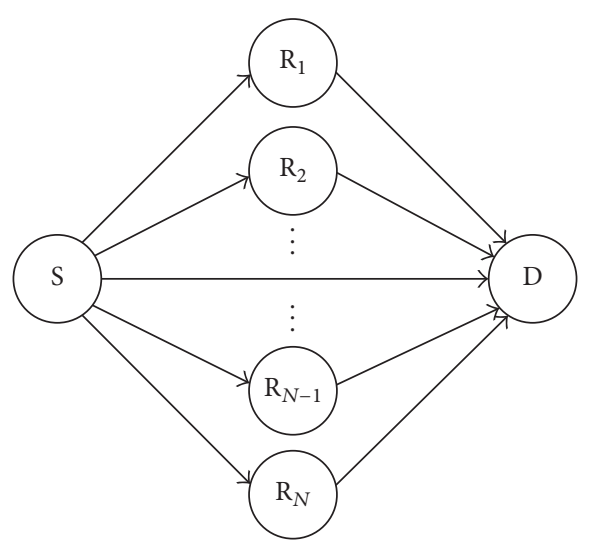

Figure 2: System model of the multiple relay communication.

successfully, and the mutual information is $I_{\mathrm{DT}}$. When $T_{1} / 2<\left|h_{s d}\right|^{2} \leq T_{1}$, the direct transmission in link S-D failed. But the retransmission is successful in the second slot, and the mutual information is $I_{\mathrm{DRT}}$. When $\left|h_{s d}\right|^{2} \leq T_{1} / 2$ and $\left|h_{s r}\right|^{2}>T_{1}$, the DF protocol is used to forward the messages and the mutual information is given as $I_{\mathrm{DF}}$. When $\left|h_{s d}\right|^{2} \leq T_{1} / 2$ and $\left|h_{s r}\right|^{2} \leq T_{1}$, the AF protocol is employed to forward the messages, and the mutual information is expressed as $I_{\mathrm{AF}}$.

\section{Multiple Relay System Model and Relay Selection Strategy}

There is a typical two-hop multirelay cooperative network shown in Figure 2, consisting of the source node $S$, the destination node $\mathrm{D}$, and $N$ relay nodes $\mathrm{R}_{i}(i=1,2, \ldots, N)$. Equipped with single omnidirectional antenna, all the above nodes operate in a half-duplex mode. Hence the entire transmission procedure is also divided into two slots, under the independent and identically distributed (i.i.d.) AWGN channel noise. In addition, all channels are also supposed to be the flat Rayleigh fading channels, with fixed channel gains and independent channel status in each transmission. The destination node can select the appropriate relay nodes and notify them to forward the source information, where the channel status information (CSI) is available between all nodes through training sequence feedback.

Based on the system model, there is a relay selection strategy as the HDAF scheme, which chooses the AF or DF scheme to forward signals adaptively according to the channel status. If the channel status of link $S-R_{i}$ is good enough for the relay to decode the source information, the DF protocol is selected to forward signals in the relay. Otherwise, the AF protocol is just used to prevent from the error propagation. According to the above strategy, the $N$ relays can be divided into two sets for comparison. The optimal relay is then selected among the $N$ relays in the premise of the maximum SNR at the destination. Finally, the specific process of the relay selection is listed as follows.

3.1. Determination of the Cooperative Relay Schemes. At first, there are some symbol definitions about the transmission power of the source and relay, respectively, that is, $P_{S 2}$ and
$P_{R 2}$, as well as the information transmission rate $R \mathrm{bit} / \mathrm{s}$. The channel noise is the AWGN with zero mean and variance $N_{0}$. Three channel parameters, such as $\left|h_{s d}\right|^{2},\left|h_{s r i}\right|^{2}$, and $\left|h_{\text {rid }}\right|^{2}$, are the channel gains of the links S-D, S-R $R_{i}$, and $\mathrm{R}_{i}-\mathrm{D}$, respectively. They are subjected to the exponential distribution with parameters of $1 / \sigma_{s d}{ }^{2}, 1 / \sigma_{\text {sri }}{ }^{2}$, and $1 / \sigma_{\text {rid }}{ }^{2}$. If the relays decode the signals from the source successfully, there will not be any interruption for transmission between source node $\mathrm{S}$ and relay node $\mathrm{R}_{i}$. For this case, the mutual information in the transmission is deduced as

$$
I_{s r i}=\frac{1}{2} \cdot \log _{2}\left(1+\frac{P_{S 2}\left|h_{s r i}\right|^{2}}{N_{0}}\right) .
$$

Equation (5) should be larger than $R$ to ensure that the transmission is not interrupted. And it can be transformed as $\left|h_{s r i}\right|^{2}>\left(2^{2 R}-1\right) N_{0} / P_{S 2}$. Thus the threshold value can be set as $T_{2}=\left(2^{2 R}-1\right) N_{0} / P_{S 2}$. When $\left|h_{s r i}\right|^{2} \geq T_{2}$, the relay can decode the signal successfully. So the DF protocol is selected to forward the signal to avoid noise amplification. When $\left|h_{\text {sri }}\right|^{2}<T_{2}$, the decoding in the relay failed. And the AF protocol is adopted to prevent error propagation.

Therefore, the candidate relays are divided into two sets according to whether successful decoding occurs or not in the relays, where the relays in set $\Omega_{\mathrm{DF}}$ select the DF scheme to forward the signals in the second slot and others in set $\Omega_{\mathrm{AF}}$ use the AF scheme. They are expressed as

$$
\begin{aligned}
& \Omega_{\mathrm{DF}}=\left\{R_{i}:\left|h_{s r i}\right|^{2}>T_{2}\right\}, \\
& \Omega_{\mathrm{AF}}=\left\{R_{i}:\left|h_{s r i}\right|^{2} \leq T_{2}\right\} .
\end{aligned}
$$

3.2. Optimal Relay Selection. Since the optimal relay means to the maximized SNR in the destination, there are two steps to obtain it. Firstly, the best relays of $R_{b}{ }^{\mathrm{DF}}$ and $R_{b}{ }^{\mathrm{AF}}$ are chosen from the sets $\Omega_{\mathrm{DF}}$ and $\Omega_{\mathrm{AF}}$, respectively. Then, the optimal relay $R_{b}$ can be chosen between relay $R_{b}{ }^{\mathrm{DF}}$ and relay $R_{b}{ }^{\mathrm{AF}}$.

For the cooperation system with the AF scheme, the destination combines the signals from the source and the relay together by the maximum ratio combination (MRC) mechanism, and the instantaneous SNR at the destination is expressed as

$$
\gamma^{\mathrm{AF}}=\gamma_{1}^{\mathrm{AF}}+\gamma_{2}^{\mathrm{AF}}
$$

where the instantaneous $\mathrm{SNR}$ is expressed as $\gamma_{1}{ }^{\mathrm{AF}}=$ $P_{S 2}\left|h_{s d}\right|^{2} / N_{0}$ in the first slot and $\gamma_{2}{ }^{\mathrm{AF}}=P_{S 2} P_{R 2}\left|h_{s r i}\right|^{2}\left|h_{\text {rid }}\right|^{2} /$ $\left[N_{0}\left(P_{S 2}\left|h_{\text {sri }}\right|^{2}+P_{R 2}\left|h_{\text {rid }}\right|^{2}+N_{0}\right)\right]$ in the second slot. Thus for the relay selection in set $\Omega_{\mathrm{AF}}$, the instantaneous $\operatorname{SNR} \gamma^{\mathrm{AF}}$ is maximized to get the best relay, so the candidate relay $R_{b}{ }^{\mathrm{AF}}$ with largest $\mathrm{SNR} \gamma_{2}{ }^{\mathrm{AF}}$ is obtained and expressed as

$$
\begin{aligned}
& R_{b}^{\mathrm{AF}} \\
& \quad=\arg \max _{R_{i} \in \Omega_{\mathrm{AF}}}\left\{\frac{P_{S 2} P_{R 2}\left|h_{s r i}\right|^{2}\left|h_{r i d}\right|^{2}}{N_{0}\left(P_{S 2}\left|h_{s r i}\right|^{2}+P_{R 2}\left|h_{r i d}\right|^{2}+N_{0}\right)}\right\} .
\end{aligned}
$$


For the cooperation system with the DF protocol, the signals from the source and the relay are combined by the MRC scheme, and the instantaneous SNR at the destination is obtained as

$$
\gamma^{\mathrm{DF}}=\min \left({\gamma_{1}}^{\mathrm{DF}}, \gamma_{2}^{\mathrm{DF}}\right)
$$

where the instantaneous SNR from the first slot is $\gamma_{1}^{\mathrm{DF}}=$ $P_{S 2}\left|h_{s r i}\right|^{2} / N_{0}$ and that of the second slot is $\gamma_{2}{ }^{\mathrm{DF}}=P_{S 2}\left|h_{s d}\right|^{2} /$ $N_{0}+P_{R 2}\left|h_{\text {rid }}\right|^{2} / N_{0}$. If all relays in set $\Omega_{\mathrm{DF}}$ can succeed in decoding the signals, the instantaneous SNR at the destination is $\gamma_{2}{ }^{\mathrm{DF}}$. Then the optimal relay $R_{b}{ }^{\mathrm{DF}}$ is represented as

$$
R_{b}^{\mathrm{DF}}=\arg \max _{R_{i} \in \Omega_{\mathrm{DF}}}\left\{\frac{P_{S 2}\left|h_{s d}\right|^{2}}{N_{0}}+\frac{P_{R 2}\left|h_{\text {rid }}\right|^{2}}{N_{0}}\right\} .
$$

Finally, the optimal relay $R_{b}$ can be chosen as the larger instantaneous SNR between $R_{b}{ }^{\mathrm{DF}}$ and $R_{b}{ }^{\mathrm{AF}}$. Then it forwards the signal from the source in the corresponding mode. And it is expressed as

$$
R_{b}=\max \left\{R_{b}{ }^{\mathrm{AF}}, R_{b}{ }^{\mathrm{DF}}\right\} .
$$

Meanwhile, the mutual information of the cooperative transmission of the HDAF scheme by the proposed relay selection strategy is

$$
I_{\mathrm{HDAF}}= \begin{cases}I_{\mathrm{DF}^{\prime}}, & \left|h_{s r b}\right|^{2} \geq T_{2} \\ I_{\mathrm{AF}^{\prime}}, & \left|h_{s r b}\right|^{2}<T_{2},\end{cases}
$$

where $\left|h_{s r b}\right|^{2}$ is the channel gain of link S- $\mathrm{R}_{b}$.

\section{Optimization of Power Allocation in the Relay Selection}

The power allocation is optimized to obtain high power efficiency, where the entire power is taken as the constraint condition and the outage probability as the objective function. Then, the outage probability of the whole cooperation system is deduced analytically. And the Lagrange multiplier method is used to solve the optimal power allocation equation.

4.1. Deduction of Outage Probability. Outage probability is defined as the probability of failure in a transmission, which is one of the most used measures to evaluate the entire wireless communications. The transmission interruption occurs when the link capacity can not attain the required user rate. In other words, the mutual information of the transmission channel is smaller than the actual transmission rate. For a single relay network in the ISHDAF scheme, the direct sourcedestination transmission or retransmission is premised on the successful decoding of the received signals in the destination. Hence, the interruption only exists in the cooperative transmission. Based on the analysis in Section 3, the outage probability of the ISHDAF scheme can be deduced as

$$
\begin{aligned}
& P_{\text {out }}^{\mathrm{ISHDAF}} \\
& \quad \operatorname{Pr}\left(\left|h_{s d}\right|^{2} \leq \frac{T_{1}}{2},\left|h_{s r}\right|^{2}>T_{1}, I_{\mathrm{DF}}<R\right) \\
& \quad+\operatorname{Pr}\left(\left|h_{s d}\right|^{2} \leq \frac{T_{1}}{2},\left|h_{s r}\right|^{2} \leq T_{1}, I_{\mathrm{AF}}<R\right) .
\end{aligned}
$$

By replacing (3) and (4) into (13), and letting $\gamma=2^{2 R}-1$, it gets

$$
\begin{aligned}
& P_{\text {out }}^{\text {ISHDAF }}=\operatorname{Pr}\left(\left|h_{s d}\right|^{2} \leq \frac{T_{1}}{2},\left|h_{s r}\right|^{2}>T_{1}, \frac{\left|h_{s d}\right|^{2} P_{S 1}}{N_{0}}\right. \\
& \left.+\frac{\left|h_{r d}\right|^{2} P_{R 1}}{N_{0}}<\gamma\right)+\operatorname{Pr}\left(\left|h_{s d}\right|^{2} \leq \frac{T_{1}}{2},\left|h_{s r}\right|^{2}\right. \\
& \quad \leq T_{1}, \frac{\left|h_{s d}\right|^{2} P_{S 1}}{N_{0}}+f\left(\frac{\left|h_{s r}\right|^{2} P_{S 1}}{N_{0}}, \frac{\left|h_{r d}\right|^{2} P_{R 1}}{N_{0}}\right) \\
& \quad<\gamma) .
\end{aligned}
$$

The probability density function (PDF) of $\left|h_{s d}\right|^{2},\left|h_{s r}\right|^{2}$, and $\left|h_{r d}\right|^{2}$ is expressed as

$$
\begin{aligned}
& f_{\left|h_{s d}\right|^{2}}(x)=\lambda_{1} e^{-\lambda_{1} x}, \\
& f_{\left|h_{s r}\right|^{2}}(x)=\lambda_{2} e^{-\lambda_{2} x}, \\
& f_{\left|h_{r d}\right|^{2}}(x)=\lambda_{3} e^{-\lambda_{3} x},
\end{aligned}
$$

$$
x>0,
$$

where $\lambda_{1}=1 / \sigma_{s d}{ }^{2}, \lambda_{2}=1 / \sigma_{s r}{ }^{2}, \lambda_{3}=1 / \sigma_{r d}{ }^{2}$. Given the exponential distribution $X$ and $Y$ with parameters $\theta_{1}$ and $\theta_{2}$, respectively, the PDF of $Z(Z=X+Y)$ is deduced by the integral equation $f_{Z}(z)=\int_{-\infty}^{\infty} f_{X}(z-y) f_{Y}(y) d y$, and it is expressed in [23] as

$$
f_{Z}(z)=\frac{\theta_{1} \theta_{2}}{\left(\theta_{2}-\theta_{1}\right)} \cdot\left(e^{-\theta_{1} z}-e^{-\theta_{2} z}\right), \quad z>0 .
$$

In addition, the probability distribution function $W$ is approximately represented as $F_{W}(x) \approx\left(\theta_{1}+\theta_{2}\right) x$ on condition of high SNR, when $W=X Y /(1+X+Y)$ [23].

Based on the above discussion, at high SNRs, the outage probability of the ISHDAF scheme can be calculated as follows:

$$
\begin{aligned}
\operatorname{Pr}\left(\left|h_{s d}\right|^{2} \leq \frac{T_{1}}{2}\right) & =1-\exp \left(-\frac{T_{1}}{2 \sigma_{s d}^{2}}\right) \\
& =1-\exp \left(-\frac{\gamma N_{0}}{2 P_{S 1} \sigma_{s d}^{2}}\right) \approx \frac{\gamma N_{0}}{2 P_{S 1} \sigma_{s d}^{2}},
\end{aligned}
$$


and similar result is obtained as $\operatorname{Pr}\left(\left|h_{s r}\right|^{2} \leq T_{1}\right) \approx \gamma N_{0} /$ $\left(P_{S 1} \sigma_{s r}^{2}\right)$.

According to (16), take $N_{0} /\left(P_{S 1} \sigma_{s d}^{2}\right)$ as $\theta_{1}$ and $N_{0} /\left(P_{R 1} \sigma_{r d}^{2}\right)$ as $\theta_{2}$; there is the following deduction:

$$
\begin{aligned}
& \operatorname{Pr}\left(\frac{\left|h_{s d}\right|^{2} P_{S 1}}{N_{0}}+\frac{\left|h_{r d}\right|^{2} P_{R 1}}{N_{0}}<\gamma\right) \\
& \quad=\int_{0}^{\gamma} \frac{\theta_{1} \theta_{2} \cdot\left(e^{-\theta_{1} z}-e^{-\theta_{2} z}\right)}{\theta_{2}-\theta_{1}} d z \\
& \quad \approx \frac{\theta_{1} \theta_{2}}{\theta_{2}-\theta_{1}} \cdot \int_{0}^{\gamma}\left[\left(1-\theta_{1} z\right)-\left(1-\theta_{2} z\right)\right] d z \\
& \quad=\frac{\theta_{1} \theta_{2}}{\theta_{2}-\theta_{1}} \cdot \int_{0}^{\gamma}\left(\theta_{2}-\theta_{1}\right) z d z=\frac{\theta_{1} \theta_{2} \gamma^{2}}{2} \\
& \quad=\frac{\gamma^{2} N_{0}^{2}}{2 P_{S 1} P_{R 1} \sigma_{s d}^{2} \sigma_{r d}^{2}} .
\end{aligned}
$$

With (18) and the descriptions mentioned above, there is

$$
\begin{aligned}
& \operatorname{Pr}\left(\frac{\left|h_{s d}\right|^{2} P_{S 1}}{N_{0}}+f\left(\frac{\left|h_{s r}\right|^{2} P_{S 1}}{N_{0}}, \frac{\left|h_{r d}\right|^{2} P_{R 1}}{N_{0}}\right)<\gamma\right) \\
& \quad=\frac{\gamma^{2}}{2} \cdot \frac{N_{0}}{P_{S 1} \sigma_{s d}^{2}}\left(\frac{N_{0}}{P_{S 1} \sigma_{s r}^{2}}+\frac{N_{0}}{P_{R 1} \sigma_{r d}^{2}}\right) .
\end{aligned}
$$

Therefore, the outage probability of a single relay ISHDAF cooperative network is expressed as

$$
\begin{aligned}
& P_{\text {out }}^{\text {ISHDAF }}=\frac{\gamma N_{0}}{2 P_{S 1} \sigma_{s d}^{2}}\left[\left(1-\frac{\gamma N_{0}}{P_{S 1} \sigma_{s r}^{2}}\right) \frac{\gamma^{2} N_{0}^{2}}{2 P_{S 1} P_{R 1} \sigma_{s d}^{2} \sigma_{r d}^{2}}\right. \\
& \left.+\frac{\gamma N_{0}}{P_{S 1} \sigma_{s r}^{2}} \frac{\gamma^{2}}{2} \frac{N_{0}}{P_{S 1} \sigma_{s d}^{2}}\left(\frac{N_{0}}{P_{S 1} \sigma_{s r}^{2}}+\frac{N_{0}}{P_{R 1} \sigma_{r d}^{2}}\right)\right] \\
& \approx \frac{\left(\gamma N_{0}\right)^{4}}{4 P_{S 1}^{2} \sigma_{s d}^{4} \sigma_{s r}^{2}}\left(\frac{1}{P_{S 1}}+\frac{\sigma_{s r}^{2}}{P_{R 1} \sigma_{r d}^{2}}\right) .
\end{aligned}
$$

Similarly, the outage probability in the HDAF relay selection strategy is denoted as

$$
\begin{aligned}
& P_{\text {out }}^{\mathrm{HDAF}}=\operatorname{Pr}\left(\left|h_{s r}\right|^{2}>T_{2}, \frac{\left|h_{s d}\right|^{2} P_{S 2}}{N_{0}}+\frac{\left|h_{r d}\right|^{2} P_{R 2}}{N_{0}}\right. \\
& <\gamma)+\operatorname{Pr}\left(\left|h_{s r}\right|^{2} \leq T_{2}, \frac{\left|h_{s d}\right|^{2} P_{S 2}}{N_{0}}\right. \\
& \left.+f\left(\frac{\left|h_{s r}\right|^{2} P_{S 2}}{N_{0}}, \frac{\left|h_{r d}\right|^{2} P_{R 2}}{N_{0}}\right)<\gamma\right) .
\end{aligned}
$$

It is obvious that $P_{\text {out }}^{\mathrm{HDAF}}$ just lacks the part of " $\left|h_{s d}\right|^{2} \leq$ $T_{1} / 2$ " when compared with $P_{\text {out }}^{\text {ISHDAF }}$. Finally, according to the above analyses, the outage probability is deduced as

$$
P_{\text {out }}^{\mathrm{HDAF}} \approx \frac{\left(\gamma N_{0}\right)^{3}}{2 P_{S 2} \sigma_{s d}^{2} \sigma_{s r}^{2}}\left(\frac{1}{P_{S 2}}+\frac{\sigma_{s r}^{2}}{P_{R 2} \sigma_{r d}^{2}}\right) .
$$

4.2. Optimization of Power Allocation. Using the Lagrange multiplier method, the optimized power allocation among the source and relay nodes to minimize the outage probability is produced as follows. For the ISHDAF scheme, with entire power as the constraint, as long as the fixed power $P$ with $P_{S 1}+P_{R 1}=P$, the optimization problem can be denoted as

$$
\begin{array}{ll}
\min & \frac{\left(\gamma N_{0}\right)^{4}}{4 P_{S 1}^{2} \sigma_{s d}^{4} \sigma_{s r}^{2}}\left(\frac{1}{P_{S 1}}+\frac{\sigma_{s r}^{2}}{P_{R 1} \sigma_{r d}^{2}}\right) \\
\text { s.t. } & P_{S 1}+P_{R 1}=P .
\end{array}
$$

Let $P_{S 1}=a_{s} P, P_{R 1}=a_{r} P$, where $a_{s}+a_{r}=1$. The Lagrange function is established as

$$
\begin{aligned}
L\left(P_{S 1}, P_{R 1}, \lambda\right)= & \frac{\left(\gamma N_{0}\right)^{4}}{4 P_{S 1}^{2} \sigma_{s d}^{4} \sigma_{s r}^{2}}\left(\frac{1}{P_{S 1}}+\frac{\sigma_{s r}^{2}}{P_{R 1} \sigma_{r d}^{2}}\right) \\
& -\lambda P\left(a_{s}+a_{r}-1\right) .
\end{aligned}
$$

Take partial derivation of (24) with respect to $a_{s}$ and $a_{r}$, respectively, and then make them equal to zero; we can get

$$
\begin{array}{r}
\frac{3}{a_{s}^{4} \sigma_{s r}^{2}}+\frac{2}{a_{s}^{3} a_{r} \sigma_{r d}^{2}}-\lambda P=0, \\
\frac{1}{a_{s}^{2} a_{r}^{2} \sigma_{r d}^{2}}-\lambda P=0 .
\end{array}
$$

By combining (25) and (26) together, and setting $e=$ $a_{s} / a_{r}$, a quadratic equation with respect to variable $e$ is obtained as

$$
e^{2}-2 e-\frac{3 \sigma_{r d}^{2}}{\sigma_{s r}^{2}}=0
$$

According to the root of (27) and $a_{s}+a_{r}=1$, the optimized solutions of $P_{S 1}$ and $P_{R 1}$ satisfying (23) are obtained, respectively, as

$$
\begin{aligned}
& P_{S 1}=P \cdot \frac{\sigma_{s r}^{4}+\sqrt{\sigma_{s r}^{2}+3 \sigma_{r d}^{2}}}{2 \sigma_{s r}^{4}+\sqrt{\sigma_{s r}^{2}+3 \sigma_{r d}^{2}}}, \\
& P_{R 1}=P \cdot \frac{\sigma_{s r}^{4}}{2 \sigma_{s r}^{4}+\sqrt{\sigma_{s r}^{2}+3 \sigma_{r d}^{2}}} .
\end{aligned}
$$

From (28) and (29), the powers $P_{S 1}$ and $P_{R 1}$ of the ISHDAF relay network in the optimized power allocation mainly depend on the channel coefficients of link S-R and link R-D, but not on that of link S-D.

Similarly, the power allocation optimization for the HDAF scheme can be defined as

$$
\begin{array}{ll}
\min & \frac{\left(\gamma N_{0}\right)^{3}}{2 P_{S 2} \sigma_{s d}^{2} \sigma_{s r}^{2}}\left(\frac{1}{P_{S 2}}+\frac{\sigma_{s r}^{2}}{P_{R 2} \sigma_{r d}^{2}}\right) \\
\text { s.t. } & P_{S 2}+P_{R 2}=P .
\end{array}
$$


Finally, the optimized powers $P_{S 2}$ and $P_{R 2}$ in the above power allocation are resolved as

$$
\begin{gathered}
P_{S 2}=P \cdot \frac{\sigma_{s r}^{4}+\sqrt{\sigma_{s r}^{2}+8 \sigma_{r d}^{2}}}{2 \sigma_{s r}^{4}+\sqrt{\sigma_{s r}^{2}+8 \sigma_{r d}^{2}}}, \\
P_{R 2}=P \cdot \frac{\sigma_{s r}^{4}}{\left(2 \sigma_{s r}^{4}+\sqrt{\sigma_{s r}^{2}+8 \sigma_{r d}^{2}}\right)} .
\end{gathered}
$$

4.3. Optimal Relay Location. The power allocation depends on the channel coefficients, which are related to the distance between the relay and the source or the destination. To obtain the maximum outage probability gain by the proposed power allocation, an optimal relay location is deduced as follows.

To simplify the analysis of power allocation, we just constrain the situations where the distance between the link S-D and the link S-R-D is approximately equal, especially when the distance is quite large. Otherwise, under the same channel noise variance $N_{0}$ in the assumed condition, the transmission of the link S-R-D is much worse than that of link S-D, which loses the sense of relay selection. And it has been adopted similarly in [21]. Then, given normalization distance of link S-D (or approximate link S-R-D) as $d_{\mathrm{SD}}=1$, and the distance $x$ of link S-R, there is $d_{\mathrm{SR}}=x$ and $d_{\mathrm{RD}}=1-x$, where $0<x<1$. When the path loss factor is considered as $\alpha=4$, the channel coefficients are obtained as $\sigma_{s r}=x^{-4}$, $\sigma_{r d}=(1-x)^{-4}$. Then, for the ISHDAF scheme, the transmit power of the source and relay is presented as

$$
\begin{aligned}
& P_{S 1}=P \cdot \frac{x^{-16}+\sqrt{x^{-8}+3(1-x)^{-8}}}{2 x^{-16}+\sqrt{x^{-8}+3(1-x)^{-8}}}, \\
& P_{R 1}=P \cdot \frac{x^{-16}}{2 x^{-16}+\sqrt{x^{-8}+3(1-x)^{-8}}} .
\end{aligned}
$$

Taking the derivation of variable $x$ in (33), it obtains

$$
\begin{aligned}
& \frac{d P_{S 1}}{d x}=P \cdot\left(\frac{16 x^{-17}}{2 x^{-16}+\sqrt{x^{-8}+3(x-1)^{-8}}}\right. \\
& \left.-\frac{\left(\left(4 x^{-9}+12(x-1)^{-9}\right) / \sqrt{x^{-8}+3(x-1)^{-8}}\right)+32 x^{-17}}{x^{16}\left(2 x^{-16}+\sqrt{x^{-8}+3(x-1)^{-8}}\right)^{2}}\right) .
\end{aligned}
$$

Equation (35) is always greater than zero in the interval of $0<x<1$. So (33) is easily recognized as the monotone increasing function since the derivation of it, (35), is greater than zero. Then, $P_{S 1}$ is kept at about $0.5 P$, when $x$ is less than 0.5 , and it tends to be $P$, when $x$ is greater than 0.9 . So the transmit power of the source is always larger than that of the relay in the proposed algorithm of the ISHDAF scheme.
Substituting (33), (34), and $\sigma_{s r}=x^{-4}, \sigma_{r d}=(1-x)^{-4}$ into (20), it gets

$$
\begin{aligned}
& P_{\text {out }}^{\mathrm{ISHDAF}}=\frac{\left(\gamma N_{0}\right)^{4} x^{8}}{4 P^{3}}(1 \\
& +\frac{x^{-16}}{x^{-16}+\sqrt{x^{-8}+3(1-x)^{-8}}} \\
& \left.+x^{8}(1-x)^{8}\left(2 x^{-16}+\sqrt{x^{-8}+3(1-x)^{-8}}\right)\right) .
\end{aligned}
$$

From (36), the systematic outage probability is relatively small in the case that the distance of link R-D is larger than that of link S-R. In other words, the relay node $\mathrm{R}$ is relatively close to the destination node $\mathrm{D}$ for better outage probability. However, when the relay node is approximately located in the middle between the source and destination node, the entire outage probability is minimal. Simultaneously, a theoretical analysis about the outage probability of the HDAF system just resembles that of the ISHDAF system. But when the relay node is close to the destination node, the outage performance decreases, and the optimal relay location closely approaches to the source node than that of the ISHDAF system. Since the order of (36) is too high to obtain the analytic solution, only numerical results are available and they will be given in the successive simulation related in Section 5.

4.4. Diversity Gain. Given the diversity gain in the proposed ISHDAF scheme, it should be divided into three cases as follows.

First, when $\left|h_{s d}\right|^{2}>T_{1}$, the source transmits directly to the destination successfully in the first time slot, and $I_{\mathrm{DT}}$ in (1) shows that the signal is transmitted only in one path. So it extracts one diversity gain in the direct transmission.

Second, the direct transmission is failed, but the retransmission is successful in the second time slot, when there is $T_{1} / 2<\left|h_{s d}\right|^{2} \leq T_{1}$. The SNR received by the destination node is twice as straightforward, while the transmission still experiences only one path. Therefore, the diversity gain is still one.

Third, the relay node starts to forward signals in the AF protocol or the DF protocol. In these two cases, the destination node receives signals from two links. So the system achieves two diversity gains in the cooperative transmission by the relay nodes. In addition, for the multirelay selection strategy under the HDAF scheme, it employs DF or AF mode to forward signals adaptively according to the channel status. Because both forward modes are required for R-D transmission, the full diversity gain of 2 is then obtained.

In summary, the proposed ISHDAF scheme obtains much more outage probability performance gain by the direct link retransmission rather than the relay forwarding, when compared with the IHDAF one in [8]. In other words, the diversity gain in the ISHDAF scheme is better than that of the IHDAF one, because the overall channel transmission effect 


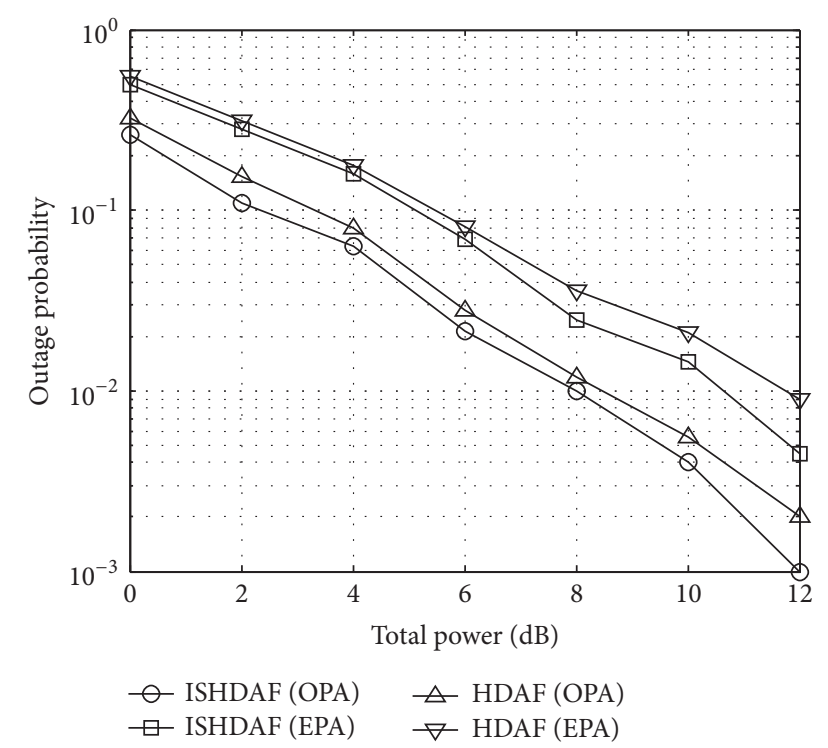

FIGURE 3: Outage probabilities between the OPA and the EPA in different forwarding strategies.

(retransmission and then cooperative relay communication) in the former is superior to that (just cooperative relay communication) in the latter.

\section{Simulation Results and Analysis}

To validate the proposed power allocation optimization algorithm for the ISHDAF and the HDAF relay selection strategy, two typical kinds of cooperative network are simulated and analyzed. For a single relay network, the outage performances by the proposed HDAF and ISHDAF strategy are compared. Besides, the optimized power allocation (OPA) and the equal power allocation (EPA) algorithms are employed in the two strategies, respectively, for comparison. In addition, for a HDAF multirelay selection network, the outage performances of the whole system with different relay numbers are simulated and compared. And the simulations for the validation of the optimal relay location are also performed in both the HDAF and the ISHDAF single relay network.

The simulation parameters are set as follows. The transmission rate is set as $R=1 \mathrm{bit} / \mathrm{s}$. The node distance is fixed as $d_{\mathrm{SD}}=1$, where $d_{i j}$ is the normalized distance between node $i$ and node $j$. All channels are Rayleigh flat fading channels, and $\alpha=4$ stands for the path loss factor. The channel noise is an AWGN with zero mean and variance $N_{0}=1$. By the Binary Phase Shift Keying (BPSK) modulation, the results are simulated and shown as follows.

Figure 3 shows the comparison of the outage probability between the OPA and the EPA scheme. They are at both the ISHDAF and the HDAF single relay network, respectively, with distance parameters of $d_{\mathrm{SR}}=0.8$ and $d_{\mathrm{RD}}=0.2$. From Figure 3, in the ISHDAF strategy, the OPA scheme achieves a much better outage performance gain over the EPA scheme. And it is also true in the HDAF strategy. Moreover, the ISHDAF strategy has larger gain over the HDAF strategy,

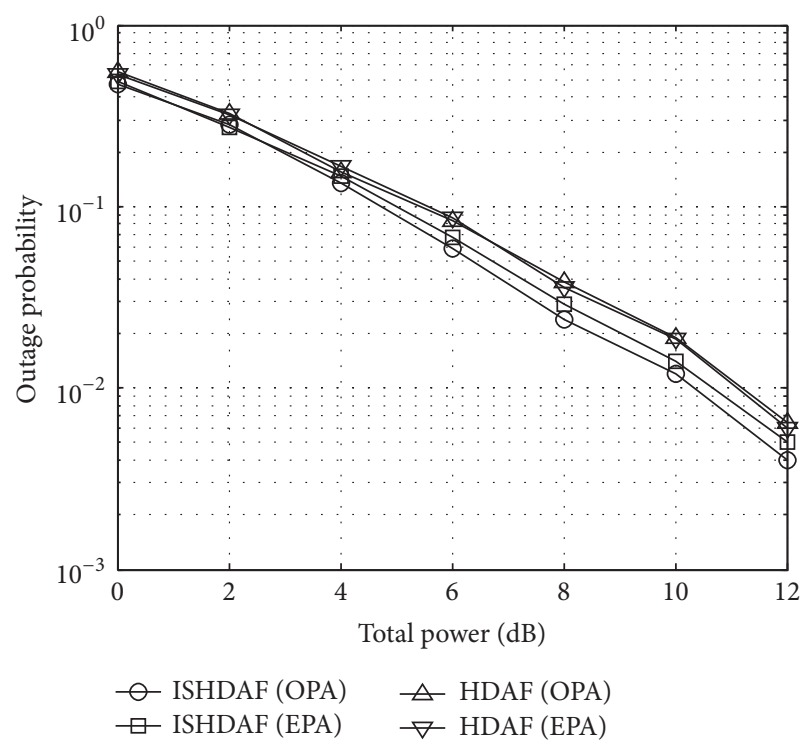

FIGURE 4: Outage probabilities between the OPA and the EPA under the specific relay location.

when they are under the same power allocation. There are nearly 0.1695 gains for the OPA scheme compared with the EPA scheme in the ISHDAF strategy and about 0.045 gains for the ISHDAF strategy compared with the HDAF strategy employed in the OPA scheme, at a total power of $2 \mathrm{~dB}$. With the increasing of the total power, the outage probability decreases, and the gains become gradually small. The reason is that when the relay node is far from the source, the outage performance of link S-R is poor. So the threshold is decreased too in the OPA scheme, which guarantees the direct transmission or retransmission in link S-D with the ISHDAF strategy. Also, from (14) to (18), there is a condition as $\left|h_{s d}\right|^{2} \leq T_{1} / 2$ for the ISHDAF to calculate the outage probability. So the outage performance of the ISHDAF scheme outperforms that of the HDAF one in the EPA scheme.

The outage performances of different schemes with the distance parameters of $d_{\mathrm{SR}}=0.2$ and $d_{\mathrm{RD}}=0.8$ are compared in Figure 4. At both the ISHDAF and the HDAF cooperation relay network, the OPA and EPA scheme have almost the same performance. Since the relay transmission opportunity increased when the relay node approaches the source node, the whole transmission in the ISHDAF scheme is similar to that of the HDAF one. And from (25) to (29), the node power based on the OPA scheme is also similar to that of the EPA scheme. At the same time, the OPA algorithm in the ISHDAF strategy achieves a better gain, when the distance of link S-R is larger than that of link R-D.

There is also a comparison of outage performance between the OPA and the EPA scheme, in the HDAF multirelay selection network. They are simulated with different number of relays, under the distance parameters of $d_{\mathrm{SR}}=0.8$ and $d_{\mathrm{RD}}=0.2$ and the results are shown in Figure 5. From Figure 5, the outage probability is reduced with the increased number of the relays. This is because many more numbers 


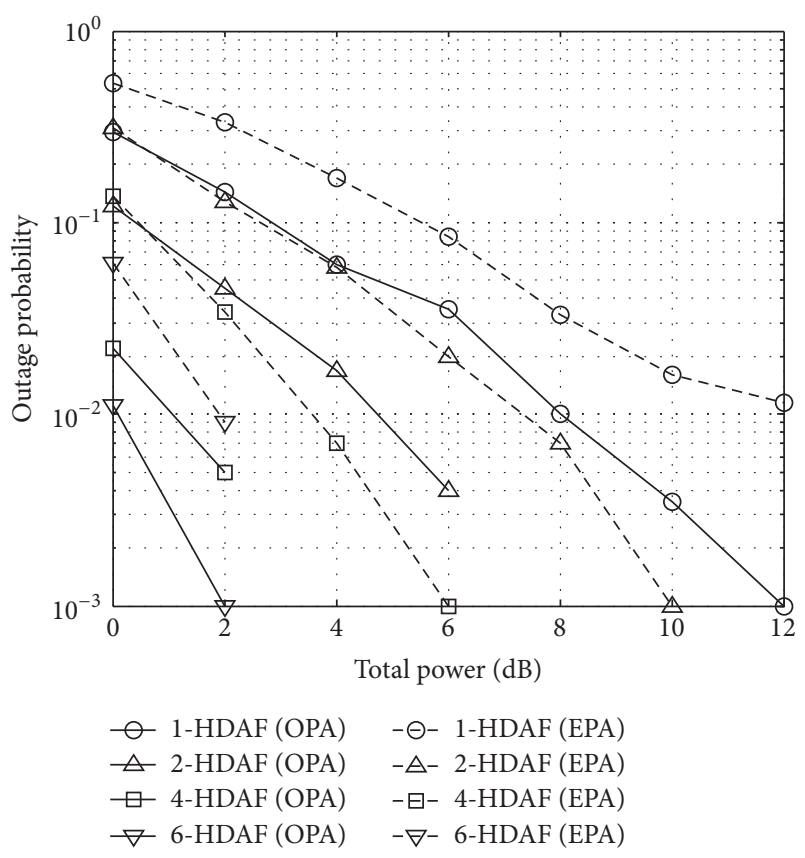

FIGURE 5: Outage probabilities among different number of the relays in the HDAF scheme.

of relays result in better channel quality of the best selected relay. It also leads to the increased mutual information in transmission; thus the outage probability of the system reduces correspondingly. In addition, Figure 5 shows that, with the same relay number in the HDAF cooperative system, OPA scheme has a significant outage performance gain than that of the EPA scheme. For instance, at a total power of $2 \mathrm{~dB}$, there are about 0.083 gains for OPA scheme when compared with those of the EPA scheme under 2 relays. Since the declined threshold results in many more opportunities for the DF protocol employed at the relay node, at such relay location, in this case, the DF protocol outperforms the AF protocol similar to that in [24].

To verify the theoretical analysis of the optimal relay location for the proposed algorithm, some simulations are performed in the cooperative single relay network. For the different relay locations, the outage probabilities of the ISHDAF and the HDAF strategies are illustrated in Figure 6, with the fixed total power of $10 \mathrm{~dB}$. From the results, the outage probability is really low when the relay node $\mathrm{R}$ is relatively close to the destination node $\mathrm{D}$. It turns out to be the lowest (i.e., best) one for the relay node $\mathrm{R}$ at the middle position between the source node $S$ and the destination node $\mathrm{D}$, which is consistent with the theoretical analysis indicated by (32). The most possible reasons mainly rely on the following reasons. When the relay is a little far from the source, the cooperative scheme at the relay performs better under the node power allocation, which is related to the link performance. Moreover, when the relay node is just in the middle between them, the performance of both link S-R and link R-D is good. The whole system can thus obtain maximum benefit in the proper power allocation ratio of the source and the relay under the proposed algorithm. In addition, the

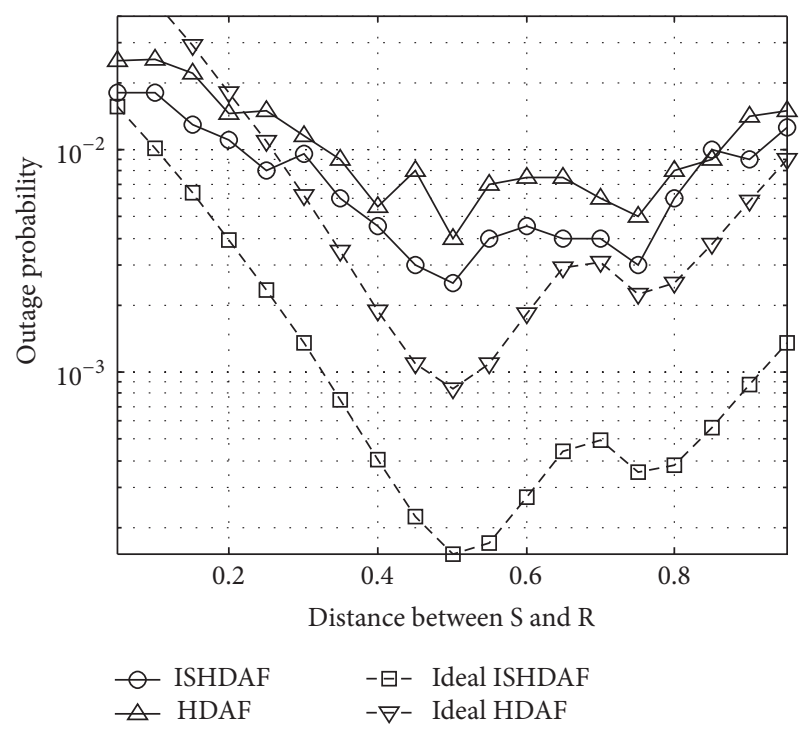

FIGURE 6: Outage probabilities among different relay locations in the OPA scheme.

simulation shows that the outage probability performance of the ISHDAF scheme is always better than that of the HDAF scheme in the proposed algorithm.

\section{Conclusion}

In this paper, an optimized power allocation algorithm is proposed, which mainly employs the two-hop single relay network with ISHDAF scheme and the multirelay selection strategy with HDAF scheme. The optimization of the proposed algorithm is just to minimize the outage probability of system under the constraints of total power of the source and relay nodes. In addition, the proposed scheme can only occupy a small amount of time complexity to obtain the power allocation optimization in a cooperative communication system. In the simulations, the proposed algorithm is applied in the ISHDAF and the HDAF scheme with the well-known three-node models, respectively. Simulation results show that the proposed algorithm can achieve much larger gain by the ISHDAF scheme than that by other ones. Also, for different number of the relay nodes in a cooperative network, the simulation comparisons show that the proposed algorithm by the HDAF relay selection strategy has a significant validity in power allocation. Simultaneously, the optimal relay location by the suggested algorithm is also established for an even better gain over current schemes. Therefore, the proposed optimized power allocation and relay location selection algorithm can be effectively adopted in cooperative IoT relay systems in practice for high power efficiency and good outage probability performance.

\section{Conflicts of Interest}

The authors declare that there are no conflicts of interest regarding the publication of this paper. 


\section{Acknowledgments}

This work was supported by the Zhejiang Provincial Natural Science Foundation of China (no. LZ14F010003, no. LY17F010019), the National Natural Science Foundation of China (no. 61471152), the Open Research Fund of National Mobile Communications Research Laboratory, Southeast University (no. 2014D02), and the Zhejiang Provincial Science and Technology Plan Project (no. 2015C31103, no. LGG18F010011).

\section{References}

[1] J.-S. Han, J.-S. Baek, S. Jeon, and J.-S. Seo, "Cooperative networks with amplify-and-forward multiple-full-duplex relays," IEEE Transactions on Wireless Communications, vol. 13, no. 4, pp. 2137-2149, 2014.

[2] P. K. Sharma and P. Garg, "Performance analysis of full duplex decode-and-forward cooperative relaying over Nakagami-m fading channels," Transactions on Emerging Telecommunications Technologies, vol. 25, no. 9, pp. 905-913, 2014.

[3] T. X. Vu, P. Duhamel, and M. D. Renzo, "Performance analysis of network coded cooperation with channel coding and adaptive DF-based relaying in Rayleigh fading channels," IEEE Signal Processing Letters, vol. 22, no. 9, pp. 1354-1358, 2015.

[4] S. S. Ikki, M. Uysal, and M. H. Ahmed, "Performance analysis of incremental-relay-selection decode-and-forward technique," in Proceedings of the 2009 IEEE Global Telecommunications Conference, GLOBECOM 2009, December 2009.

[5] T. T. Duy and H. Y. Kong, "On performance evaluation of hybrid decode-amplify-forward relaying protocol with partial relay selection in underlay cognitive networks," Journal of Communications and Networks, vol. 16, no. 5, pp. 502-511, 2014.

[6] Q. F. Zhou and F. C. M. Lau, "Two incremental relaying protocols for cooperative networks," IET Communications, vol. 2, no. 10, pp. 1272-1278, 2008.

[7] J. Wu, D. He, J. Bao, X. Xu, and B. Jiang, “Optimization of power allocation and outage probability of cooperative relay networks," in Proceedings of the 8th International Conference on Wireless Communications and Signal Processing, WCSP 2016, October 2016.

[8] Z. Bai, J. Jia, and C. X. Wang, "Performance analysis of SNRbased incremental hybrid decode-amplify-forward cooperative relaying protocol," IEEE Transactions on Communications, vol. 63, no. 6, pp. 2094-2106, 2015.

[9] L. Wu, Y. Wang, J. Han et al., "Optimal power allocation for wireless uplink transmissions using successive interference cancellation," KSII Transactions on Internet and Information Systems, vol. 10, no. 5, 2016.

[10] K. K. Gurrala and S. Das, "Minimized ser based power allocation for multi HDAF relay cooperative network using differential evolution algorithm," in Proceedings of the 11th IEEE India Conference, INDICON 2014, December 2014.

[11] H. Xiao and S. Ouyang, "Power allocation for a hybrid decodeamplify-forward cooperative communication system with two source-destination pairs under outage probability constraint," IEEE Systems Journal, vol. 9, no. 3, pp. 797-804, 2015.

[12] A. Kwolek-Folland, "Power allocation for two source destination pair cooperative communication system under the usage probability constraint," Journal of the Japanese Association for Petroleum Technology, vol. 4, no. 2, pp. 74-83, 2015.
[13] Z.-K. Zhou and Q. Zhu, "Joint power allocation and multi-relay selection scheme based on system outage probability," Journal of China Universities of Posts and Telecommunications, vol. 21, no. 5, pp. 9-16, 2014.

[14] M. Qian, C. Liu, Y. Fu, and W. Zhu, "A relay selection and power allocation scheme for cooperative wireless sensor networks," KSII Transactions on Internet and Information Systems, vol. 8, no. 4, pp. 1390-1405, 2014.

[15] T. T. Duy and H.-Y. Kong, "Performance analysis of hybrid decode-amplify-forward incremental relaying cooperative diversity protocol using SNR-based relay selection," Journal of Communications and Networks, vol. 14, no. 6, pp. 703-719, 2012.

[16] Y. Xing, Y. Chen, C. Lv, Z. Gong, and L. Xu, "Swarm intelligencebased power allocation and relay selection algorithm for wireless cooperative network," KSII Transactions on Internet and Information Systems, vol. 10, no. 3, pp. 1111-1130, 2016.

[17] H. Wang and Q. Zhu, "Power Allocation Scheme based on System Outage Probability for Energy-Harvesting Cooperative Networks," in Proceedings of the 2015 4th National Conference on Electrical, Electronics and Computer Engineering, pp. 617-622, Xi'an, China, December 2015.

[18] Q. Zhang, Z. Feng, T. Yang, and W. Li, "Optimal power allocation and relay selection in multi-hop cognitive relay networks," Wireless Personal Communications, vol. 86, no. 3, pp. 1673-1692, 2016.

[19] J. Zhang, J. Jiang, J. Bao, B. Jiang, and C. Liu, "Improved relay selection strategy for hybrid decode-amplify forward protocol," Journal of Communications, vol. 11, no. 3, pp. 297-304, 2016.

[20] M. H. D. Khan and M. S. Elmusrati, "Performance analysis of power allocation and relay location in a cooperative relay network," in Proceedings of the 17th IEEE International Conference on Advanced Communications Technology, ICACT 2015, pp. 444-449, July 2015.

[21] L. Han, J. Mu, S. Liu et al., "Optimization of power allocation and relay location for decode-and-forward relaying in the presence of co-channel interference," in Proceedings of the Third International Conference on Communications, Signal Processing, and Systems, pp. 319-327, Springer International Publishing, 2015.

[22] Y. W. P. Hong, W. J. Huang, and C. C. J. Kuo, Cooperative Communications and Networking, Cambridge University Press, Cambridge, UK, 2009.

[23] W. Qingtao, "Research on outage probability in relay systems," Computer Engineering and Applications, vol. 49, no. 11, pp. 2426, 2013.

[24] O. J. Pandey, A. Trivedi, and M. K. Shukla, "Outage performance of decode-forward and amplify-forward protocols in cooperative wireless communication," in Proceedings of the 10th IEEE and IFIP International Conference on Wireless and Optical Communications Networks, WOCN 2013, pp. 1-5, July 2013. 


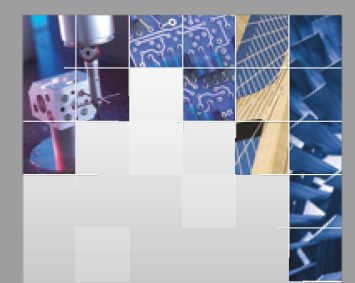

\section{Enfincering}
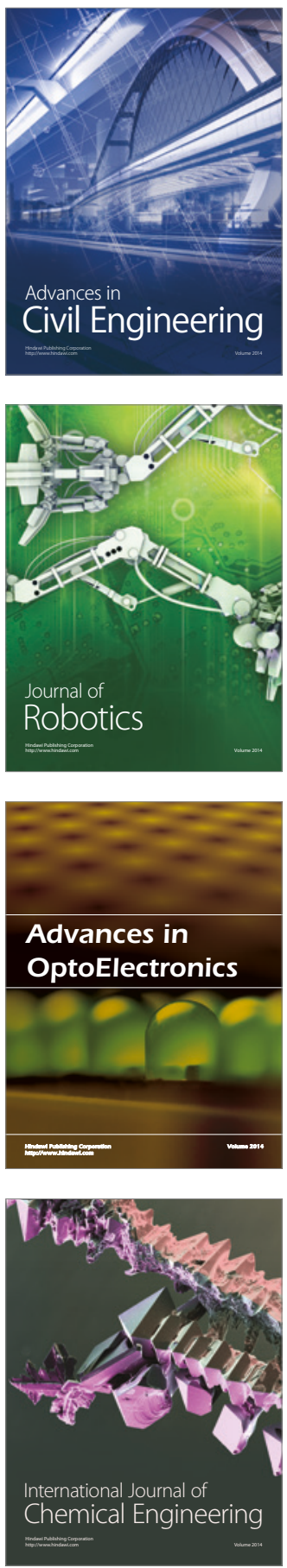

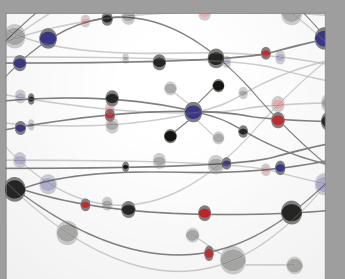

The Scientific World Journal

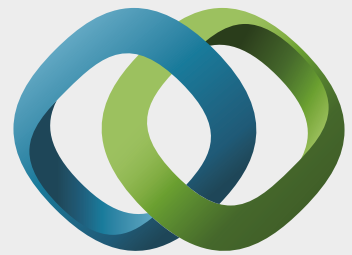

\section{Hindawi}

Submit your manuscripts at

https://www.hindawi.com
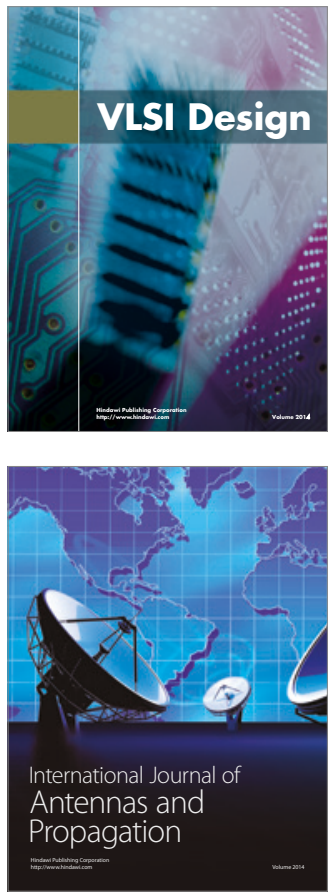

\section{Rotating}

Machinery
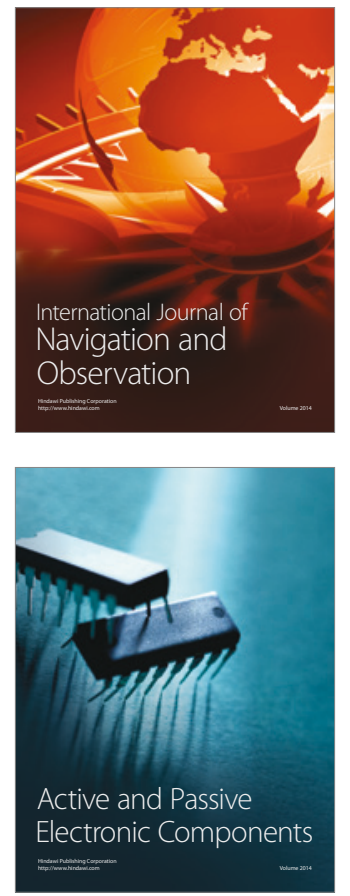
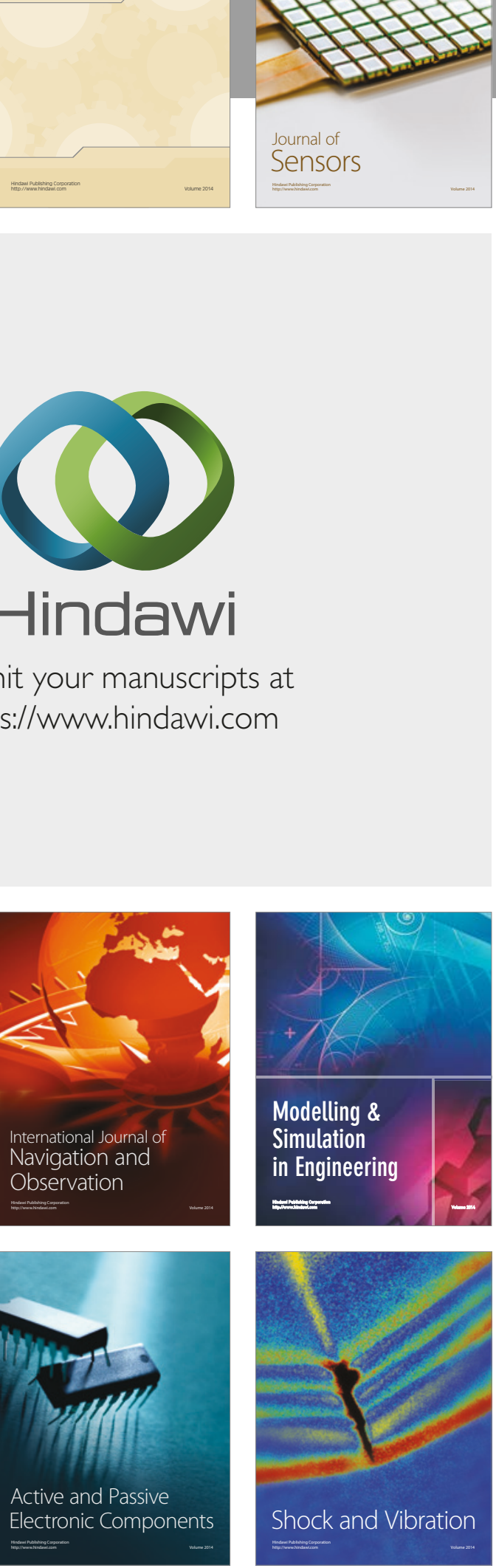
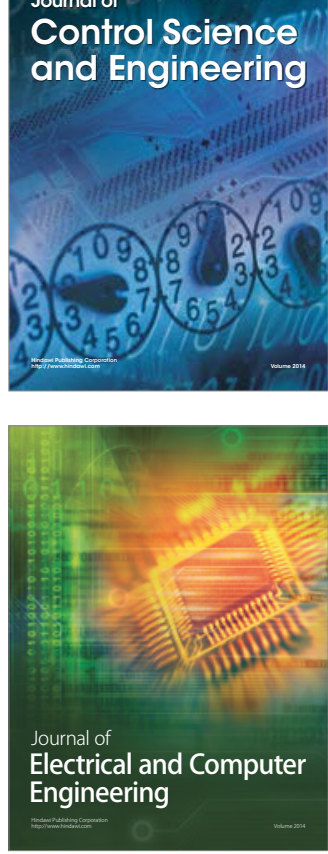

Distributed

Journal of

Control Science

and Engineering
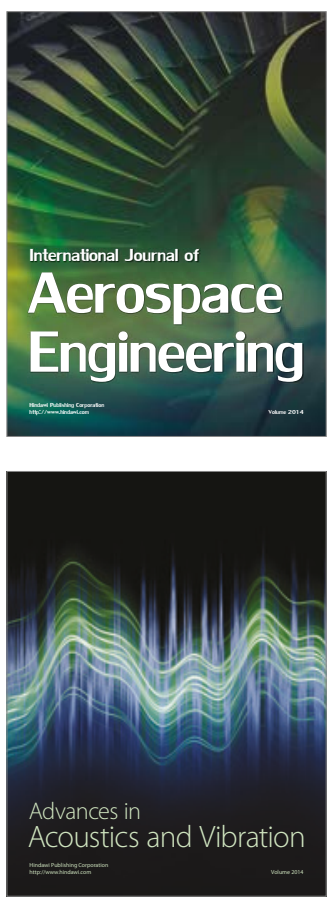

Sensor Networks 